\title{
La formation des maîtres : débats et perspectives
}

Introduction

\section{Alain Boissinot}

\section{CpenEdition}

\section{Journals}

Édition électronique

URL : http://journals.openedition.org/ries/920

DOI : $10.4000 /$ ries.920

ISSN : 2261-4265

Éditeur

Centre international d'études pédagogiques

Édition imprimée

Date de publication : 1 décembre 2010

Pagination : 27-36

ISBN : 978-2-8542-583-1

ISSN : $1254-4590$

Référence électronique

Alain Boissinot, «La formation des maîtres : débats et perspectives », Revue internationale d'éducation de Sèvres [En ligne], 55 | décembre 2010, mis en ligne le 01 septembre 2011, consulté le 30 avril 2019 URL : http://journals.openedition.org/ries/920 ; DOI : 10.4000/ries.920 


\title{
Former des enseignants
}

\section{Introduction}

\section{La formation des maîtres : débats et perspectives}

\begin{abstract}
Alain Boissinot
$\mathrm{Au}$ moment où la France entreprend un réagencement en profondeur de son système de formation des maîtres, il paraît opportun d'élargir le champ du débat et d'étudier la façon dont des pays de tradition différente s'organisent en ce domaine. Disons-le d'entrée : si d'aucuns pouvaient espérer trouver dans tel ou tel pays un modèle stabilisé dont il soit possible de s'inspirer, l'examen vient décevoir cette attente. Ce qui frappe au contraire, c'est que partout la formation des maîtres, qui est considérée comme un enjeu majeur et tend le plus souvent à être allongée, fait l'objet de remaniements successifs et de plans de réforme. Elle est - logiquement - régulièrement remise en cause chaque fois que les résultats d'un système éducatif paraissent insuffisants, ou en tout cas en deçà d'enjeux mondialisés devenus prégnants. On notera aussi que, comme il arrive souvent, la France semble vouloir explorer telle ou telle option au moment même où d'autres pays, qui l'ont expérimentée plus tôt, se livrent à un réexamen critique de ses conséquences...

La confrontation des organisations retenues par des pays très divers, si elle ne permet pas de dégager un modèle, aide en revanche à identifier des problématiques récurrentes, autour desquelles se développent les débats et par rapport auxquelles, au fil des réformes, se déplacent les points d'équilibre. De ce point de vue, les polémiques que connaît notre pays paraissent moins "franco-françaises » qu'on ne le dit parfois. On essaiera ci-dessous de signaler les principaux nœuds problématiques autour desquels se distribuent les interrogations. Bien sûr, ces problématiques interfèrent sans cesse entre elles et ne sont ici distinguées que pour la clarté de l'exposé.
\end{abstract}

\section{Problématiques récurRentes}

\section{Savoirs et professionnalisation}

Une première tension, que connaissent tous les systèmes de formation, est celle qui oppose une démarche référée aux disciplines et aux savoirs théoriques, et une volonté de professionnalisation. 
La situation du Québec, on le verra dans l'article d'Y. Lenoir, est de ce point de vue significative. Dans les années soixante, en même temps que s'universitarise la formation des maîtres, s'amorce le passage d'une conception humaniste d'origine européenne à des orientations plus professionnalisantes, voire instrumentales. Ce débat croise celui qui porte sur les rôles respectifs des facultés disciplinaires et des facultés d'éducation, l'entrée professionnalisante s'affirmant plus facilement dans le primaire que dans le secondaire, plus étroitement lié à la tradition disciplinaire. Ligne de fracture qu'on retrouve bien sûr en France: E. Fraisse rappelle que les IUFM, dès leur naissance, ont échoué à rapprocher en profondeur les logiques du premier et du second degré. Au Québec, à partir des années quatre-vingt-dix, les réformes successives mettent l'accent sur les exigences professionnalisantes en posant le principe d'un "référentiel de compétences »: on y reviendra ci-dessous.

Les différents pays tentent le plus souvent explicitement d'articuler les diverses composantes de la formation: B. Alen montre comment la relation entre savoir général et méthodologie de l'enseignement structure le système argentin, et comment les réformes les plus récentes tentent de combiner formation générale, formation spécifique pour un niveau éducatif donné, formation par la pratique professionnelle. On retrouve en Suède ou aux États-Unis des approches comparables. L'affirmation de la dimension professionnalisante renvoie à une question présente dans la plupart des articles proposés ici, comme dans la réforme actuellement menée en France, celle des stages et des partenariats permettant la formation en milieu professionnel (voir par exemple l'article d'O. McNamara) : approche qui pourrait déboucher sur des modèles de formation en alternance.

Un aspect particulier de la professionnalisation est envisagé par divers pays notamment lorsqu'ils font face à une pénurie d'enseignants : non pas professionnaliser mais recruter des professionnels, en validant des compétences acquises (voir les exemples de l'Argentine ou des États-Unis). La France n'ignore pas cette dimension du recrutement, et a toujours ménagé, à côté des recrutements par concours, une place pour un appel à des personnels contractuels ou vacataires, notamment en matière d'enseignements professionnels.

\section{L'approche par les compétences}

Dès lors que l'on aborde la formation des maîtres non plus seulement en fonction des savoirs à enseigner (approche disciplinaire) mais du point de vue des savoir-faire attendus des futurs enseignants, on est logiquement conduit à se poser la question d'un "référentiel de compétences » : c'est sans doute l'un des points sur lesquels le débat est le plus vif.

Plusieurs articles évoquent les procédures retenues dans la période récente. Y. Lenoir décrit le référentiel mis en place au Québec par la réforme de 2001. La 
façon dont se déclinent les douze compétences, jusqu'à la reconnaissance d'une dimension éthique dans l'exercice de la profession enseignante, paraîtra sans doute familière au lecteur français ( $c f$. infra). Comme le montre A. L. Goodwin pour les États-Unis, la définition des compétences attendues par le futur employeur est d'autant plus nécessaire comme élément de cohérence dans un système où la formation elle-même est largement décentralisée, voire privatisée. Les deux témoignages sur la situation du Royaume-Uni montrent comment elle a partie liée avec des procédures d'évaluation soucieuses d'apprécier, selon une problématique désormais prégnante, l'efficience des processus mis en œuvre. L'identification des compétences dialogue en effet avec celle d' " indicateurs de performance ", participant d'une culture rationaliste voire technocratique dont plusieurs articles signalent qu'elle peut paraître peu compatible avec les conceptions humanistes traditionnelles de l'enseignement. L'article d'A. Théodoropoulou et A. Théodoridis exprime de façon particulièrement radicale cette inquiétude, critiquant les réorganisations entreprises en Grèce sous l'influence européenne au nom d'une philosophie globale de l'éducation.

\section{Le rôle de l'État}

Une autre problématique, évidemment liée à l'histoire politique et administrative des différents pays, concerne le niveau de responsabilité en matière de formation des maîtres : contrôle de l'État, décentralisation et/ou déconcentration plus ou moins marquées, dans un même pays le point d'équilibre se déplace souvent au fil des réformes.

La tendance dominante est au renforcement du contrôle de la formation des maîtres par l'État central, préoccupé souvent de résultats du système éducatif jugés insuffisants à l'aune des comparaisons internationales et des enjeux de la mondialisation. Cela n'étonne pas en France, où, comme le rappelle E. Fraisse, la formation des maîtres est l'affaire de l'État, et devient à l'occasion une affaire d'État. Y. Lenoir montre comment, au Québec, la réforme de 1994 marque la mainmise du ministère de l'Éducation sur les programmes de formation, tendance renforcée en 2001 par la diffusion d'un nouveau référentiel de compétences. De la même façon, en Argentine, la Loi d'éducation nationale de 2006 (LEN) s'accompagne de la création d'un Institut national de formation des enseignants (INFD). O. Mc Namara montre comment l'Angleterre connaît depuis les années quatre-vingt un mouvement de centralisation et de contrôle politique croissant sur la formation des enseignants, évolution d'autant plus notable qu'elle se poursuit sous des gouvernements conservateurs puis travaillistes.

Mais des pays de tradition beaucoup plus décentralisée connaissent les mêmes tendances. Ainsi, aux États-Unis, malgré une tradition de forte subsidiarité au profit des États, voire du niveau local, le gouvernement fédéral pèse très fortement en agissant notamment sur les financements : A. L. Goodwin analyse 
ce mécanisme à partir de l'exemple de l'État de New York. Cette tension est également le fil conducteur de l'article de P. Klingbjer : le gouvernement suédois renforce et réorganise la formation des maîtres en une démarche qui semble contradictoire avec la décentralisation de l'enseignement supérieur.

\section{Le choix des opérateurs}

La question du niveau d'initiative et de responsabilité croise logiquement celle du choix des opérateurs en matière de formation des maîtres : structures spécifiques (écoles normales, IUFM...), universités, voire organismes privés dans le cadre d'un cahier des charges?

Dans la plupart des pays ici étudiés, on constate le même mouvement, au cours des trente dernières années du vingtième siècle, vers l'universitarisation de la formation des maîtres. P. Klingbjer montre comment la Suède, dès les années soixante-dix, est passée «d'une formation purement professionnelle à une formation universitaire à la fois disciplinaire et professionnalisante. » Le constat est le même en Angleterre ou dans les Balkans, comme l'indique Pavel Zgaga. Au Québec, la suppression des écoles normales en 1969 ouvre un processus identique, l'université étant responsable de la formation des maîtres sur la base d'un cahier des charges. Il est intéressant de noter que le débat entre formation théorique et professionnalisation passe alors à l'intérieur de l'université, où s'établit une tension entre facultés disciplinaires et facultés d'éducation. L'Argentine, marquée par la tradition du «normalisme », représente à cet égard une situation un peu particulière : B. Alen montre le rôle des instituts supérieurs de formation des enseignants, chapeautés depuis 2007 par un Institut national, qui constituent dans de nombreux points du territoire l'unique offre d'enseignement supérieur. Comme le soulignent la plupart des auteurs, l'universitarisation de la formation des maîtres est un enjeu essentiel pour permettre le lien avec la recherche.

On reconnaît dans les différents éléments de cette problématique les principaux ingrédients du débat français, rappelé par E. Fraisse, et qui aboutit, avec un temps de retard sur la plupart des autres pays, à l'affirmation récente de l'intégration de la formation des maîtres dans l'université. La tradition des écoles normales, le poids de la distinction entre premier et second degré, expliquent ce retard. Avec un peu de recul, on voit bien comment les IUFM (et on se souvient des débats qui ont entouré la reconnaissance, fragile, de leur caractère universitaire) ont constitué de façon transitoire une solution de compromis entre la logique des écoles normales et celle des universités. Enfin, avec l'exemple américain analysé par A. L. Goodwin, on voit se dessiner une autre perspective : les autorités publiques choisissant d'agir par le biais de la définition d'un cahier des charges et par des appels d'offre, il devient possible d'envisager la privatisation de processus de formation des maîtres, notamment dans le domaine de la professionnalisation. 


\section{Formation et recrutement}

La question de la définition du niveau de responsabilité en matière de formation rejoint celle de l'articulation entre processus de formation et procédure de recrutement: le cas français illustre bien les ambiguïtés qui existent souvent en ce domaine.

Comme le montre E. Fraisse, il s'agit de gérer «la coexistence de deux logiques distinctes : celle de la délivrance d'un grade par l'université, celle du recrutement par l'employeur». On sait comment, en France, la récente " masterisation " de la formation des maîtres a immédiatement posé le problème du statut et de la place des concours de recrutement. Jusque-là, le CAPES et plus encore l'agrégation fonctionnaient de fait à la fois comme reconnaissance d'un niveau de formation et comme procédure de recrutement, selon les traditions de la fonction publique française. Désormais, c'est le master qui a vocation à garantir le niveau de formation, à la fois théorique et professionnel. Quand on sait à quel point les épreuves des concours modélisaient la représentation des différentes disciplines, on mesure combien la réforme déstabilise les fonctionnements traditionnels. En témoigne par exemple l'émoi qui a saisi certains membres de jurys à l'idée de voir apparaître dans les épreuves du concours une vérification de la compétence définie par le Cahier des charges de la formation des maîtres comme "Agir en fonctionnaire de l'État et de façon éthique et responsable ». Le débat à ce sujet n'aurait en effet lieu d'être que si les épreuves du concours engageaient la définition de la discipline : or cela est désormais le rôle du master, le concours servant à apprécier l'employabilité, sur une fonction précise, du titulaire de ce diplôme. Comment s'étonner dès lors que le futur employeur veuille s'assurer de l'acceptation par le candidat de certains principes de base de son futur métier?

La distinction entre reconnaissance de la qualification et processus de recrutement ouvre bien sûr la voie à diverses procédures possibles : on verra par exemple, dans l'article d'Y. Lenoir, comment les commissions scolaires du Québec recrutent le personnel enseignant, et comment coexistent différentes formes d' " autorisation d'enseigner ». La situation américaine pose aussi très clairement la question de l'existence, à côté des modalités classiques de formation des maîtres, d'une reconnaissance de "parcours alternatifs».

\section{Perspectives}

À la lumière des ces confrontations, il est tentant de revenir sur certains débats soulevés par les réformes entreprises aujourd'hui en France.

\section{Le Cahier des charges français de la formation des maîtres}

Les expériences étrangères signalent bien le caractère à la fois incontournable et périlleux du débat sur les compétences. En France, la première tentative pour expliciter les attentes de l'institution en ce domaine est la circulaire publiée 
en mai $1997^{1}$, qui tente de définir l'exercice du métier d'enseignant en référence à la fois aux cadres du système éducatif, de la classe et de l'établissement. L'étape suivante est la publication dix ans après du Cahier des charges de la formation des maîtres en $I U F M^{2}$, repris à quelques détails près, pour ce qui est des dix compétences attendues, par l'arrêté du 12 mai $2010^{3}$.

Si on les lit en tant que référentiels pour la formation des maîtres, les textes publiés en 1997 et en 2007 puis 2010 paraissent très convergents, comme en témoigne le tableau suivant, qui met en parallèle les chapitres de la circulaire de 1997 et les dix compétences du «cahier des charges».

\begin{tabular}{|l|l|l|}
\hline \multicolumn{2}{|c|}{1997} & \multicolumn{1}{c|}{2007 et 2010} \\
\hline $\begin{array}{l}\text { Exercer sa responsabilité } \\
\text { au sein } \\
\text { du système éducatif }\end{array}$ & $\begin{array}{l}\text { - Agir en fonctionnaire de l'État et de façon } \\
\text { éthique et responsable }\end{array}$ \\
\hline $\begin{array}{l}\text { Exercer sa responsabilité } \\
\text { dans la classe }\end{array}$ & 1. Connaître sa discipline & $\begin{array}{l}\text { - Maîtriser les disciplines } \\
\text { et avoir une bonne culture générale } \\
\text { - Maîtriser la langue française } \\
\text { pour enseigner et communiquer } \\
\text { - Se former et innover }\end{array}$ \\
\hline Id. & $\begin{array}{l}\text { 2. Savoir construire } \\
\text { des situations } \\
\text { d'enseignement } \\
\text { et d'apprentissage }\end{array}$ & $\begin{array}{l}\text { - Concevoir et mettre en oeuvre } \\
\text { son enseignement } \\
\text { - Évaluer les élèves } \\
\text { - Maîtriser les TIC }\end{array}$ \\
\hline Id. & 3. Savoir conduire la classe & $\begin{array}{l}\text { - Organiser le travail de la classe } \\
\text { - Prendre en compte la diversité des élèves }\end{array}$ \\
\hline $\begin{array}{l}\text { Exercer sa responsabilité } \\
\text { dans l'établissement }\end{array}$ & $\begin{array}{l}\text { - Travailler en équipe et coopérer } \\
\text { avec les parents et les partenaires de l'école }\end{array}$ \\
\hline
\end{tabular}

Cette continuité ne doit pas occulter le fait que l'arrêté publié en 2007 introduit un certain nombre de nouveautés s'agissant aussi bien des contenus que de la méthode.

Présenté explicitement comme un référentiel de compétences destiné aux maîtres du premier et du second degré, le texte est plus solidement structuré et plus détaillé que celui de 1997. Reprenant une approche qui avait été mise au point lors de l'élaboration du Socle commun de connaissances et de

\footnotetext{
1. «Mission du professeur exerçant en collège, en lycée d'enseignement général et technologique ou en lycée professionnel », Direction des lycées et collèges-Direction générale de l'enseignement supérieur, 23 mai 1997.

2. Arrêté du 19 décembre 2006 publié dans les premiers jours de 2007. J'ai retracé l'histoire de ces textes dans un article paru dans la revue Administration et Éducation, $\mathrm{n}^{\circ}$ 4, 2008.

3. L'arrêté de 2010 n'introduit que des variantes légères. Il prend en compte certains dispositifs liés à des réformes récentes, comme celle des lycées ; il insiste sur le développement des technologies numériques et sur la prévention de la violence.
} 
compétences (juillet 2006), il décline chaque compétence en connaissances, capacités et attitudes. Comme dans le cadre du socle commun, cette présentation a le grand mérite de neutraliser l'opposition gênante entre savoirs et savoirfaire et d'éviter les inutiles conflits de priorité entre connaissances théoriques et pédagogie.

D'autre part, le Cahier des charges prend en compte des préoccupations qui se sont affirmées depuis 1997 et/ou qui avaient été sous-estimées à l'époque. On relèvera notamment l'accent mis sur la pratique d'au moins une langue vivante étrangère, sur l'orientation et la connaissance du monde professionnel, le renouvellement de la problématique de la laïcité, écho des débats récents, et surtout l'insistance sur la maîtrise des technologies numériques.

$\mathrm{Si}$, en matière de contenus, la continuité l'emporte largement, en revanche le statut du texte paru en 2007 est beaucoup plus clair. Alors que la circulaire de 1997, émanant de deux directions pédagogiques, n'engageait pas la direction des écoles ni celle des personnels, l'arrêté de 2007, signé par les deux ministres en charge de l'enseignement scolaire et de l'enseignement supérieur, couvre cette fois l'ensemble du champ, premier degré compris (entre temps d'ailleurs la direction des lycées et collèges est devenue direction de l'enseignement scolaire). Le transfert de responsabilité en matière de formation vers les universités étant largement amorcé par la loi d'orientation d'avril 2005, il s'agit désormais de garantir le droit de regard de l'Etat-employeur, et d'assurer la cohérence nationale du dispositif qui se met en place : «La formation des maîtres est une éminente responsabilité que l'Etat républicain vient de confier aux universités. Il lui appartient d'en garantir la qualité sur l'ensemble du territoire national ». Du coup le texte peut s'affirmer à la fois comme "référentiel de compétences» pour les enseignants (l'expression est cette fois assumée), et comme «cahier des charges " pour l'opérateur de formation. Pour sa part, l'arrêté du 12 mai 2010 est signé à la fois par le secrétaire général du ministère et par le directeur général de l'enseignement scolaire ${ }^{4}$.

Le cahier des charges ainsi défini permettra-t-il d'éviter le reproche de technicisme et d'utilitarisme que rencontrent beaucoup d'expériences étrangères $?^{5}$ La réponse dépendra sans doute largement de l'usage qui en sera fait. Le risque est de le confondre avec un programme de formation, au lieu de l'utiliser comme un moyen d'apprécier, du point de vue des pratiques professionnelles, les effets de celle-ci. Il ne devrait pas s'agir de «bachoter " pour

4. Mais il n'engage pas le ministère de l’Enseignement supérieur et de la Recherche, qui entre temps est devenu un ministère indépendant.

5. Dans un entretien publié le 30 avril 2010 par l'AEF, Shirley Lawes, professeur en langues vivantes à l'Université de Londres, déclarait : « En Angleterre, l'approche par compétences montre ses limites (...) L’État a défini et imposé aux universités un corpus de compétences très strict sur la base duquel sont évalués les étudiants au moment du Post graduate certificate in education. C'est une formation très rigide et utilitariste tournée vers les résultats, les standards. Or, être professeur ne se limite pas à observer et à reproduire des gestes ; devenir enseignant nécessite d'abord d'acquérir une distance critique par rapport à sa discipline, et la manière d'exercer son métier. ". De nombreux articles réunis dans ce numéro expriment la même inquiétude. 
elles-mêmes les compétences ainsi définies, mais simplement de vérifier si la formation a permis de les développer de façon satisfaisante. Autrement dit, la "définition des compétences" ne dispense pas d'une réflexion sur les disciplines d'enseignement, mais doit s'y rajouter. Or, il semble souvent que cette réflexion sur les disciplines reste un angle mort du débat entre formation théorique et professionnalisation.

\section{La dimension didactique}

Comme le souligne E. Fraisse, la question des savoirs nécessaires aux maîtres est fréquemment abordée de façon abusivement simplificatrice : "On réduit trop souvent cette question à une simple opposition entre les connaissances académiques (la science, le savoir) et la pédagogie, réduite à une idéologie ou à une série de recettes pratiques et comportementales de bon sens face à une classe ou un groupe d'élèves. " Les médias usent et abusent de ce manichéisme, orchestrant de façon répétitive des affrontements entre les tenants supposés des deux extrêmes : Meirieu contre Finkielkraut naguère, Descoings contre Brighelli pour tenter de renouveler le genre... On passe ainsi complètement à côté du vrai débat, qui engage la définition des disciplines et leur organisation didactique. Or c'est sans doute du fait de malentendus en ce domaine que surviennent les principales difficultés des jeunes enseignants et que leur formation paraît inadaptée.

Les témoignages des débutants sont à ce sujet révélateurs. Peu après la rentrée scolaire 2010, le quotidien Le Monde consacre une page à la situation d'une jeune enseignante de français dans un collège de l'académie d'AixMarseille $^{6}$. Parmi les préoccupations qu'elle exprime, plusieurs relèvent bien de la gestion de la classe et du savoir-faire pédagogique. Mais visiblement la difficulté principale est ailleurs, et réside dans l'inadéquation entre la représentation de la discipline qui s'est forgée pendant les études universitaires et les besoins des élèves : "Avec mes cinquièmes, on va travailler la "chanson". Je suis enthousiaste, c'était mon sujet de master. Je leur explique qu'on va partir du Moyen Age, pour arriver aux chansons actuelles. On se lance avec un texte très court de Guillaume de Machaut. Mais, impossible d'avancer, il y a trop de bruit. Je mets des mots, je punis, aucun effet. Alors j'arrête mon cours et annonce une punition générale : une dictée. Dans l'empressement, je choisis un texte de La Fontaine ( La Fille), bien trop difficile. C'est la catastrophe. Je mets neuf zéros... ». On voit bien ici que l'enjeu n'est pas pédagogique : aucune formation sur la "tenue de classe ", théorique ou pratique, à l'université ou en "compagnonnage », ne résoudrait le problème. Celui-ci est clairement didactique : peut-on transférer directement un travail universitaire en cours de cinquième ? Pour travailler sur la chanson, faut-il partir des « classiques » pour aller vers la période

6. Le Monde, 9 octobre 2010, « Le journal angoissé d'une prof débutante ». 
contemporaine ou l'inverse? Comment articuler le travail sur les textes et l'apprentissage de l'orthographe pour que la dictée n'apparaisse pas seulement comme une punition? Il serait aisé de multiplier les exemples de ce type, dans divers champs disciplinaires.

L'un des bénéfices que l'on peut attendre de la réforme confiant aux universités la formation des maîtres serait de sensibiliser celles-ci à ces problématiques didactiques. Quels sont les savoirs théoriques effectivement nécessaires pour enseigner les disciplines scolaires? Comment au besoin faire évoluer la définition de ces disciplines pour tenir compte à la fois des évolutions de la science et de celles des publics visés ? Selon quelles progressions et grâce à quelles démarches sont-elles assimilables par les élèves ? Plutôt que de rejouer à l'infini la querelle entre savoirs et pédagogie, c'est sans doute en abordant clairement ces questions que l'on améliorera la formation des maîtres. Il y a là un enjeu majeur pour une Université désormais consciente de sa responsabilité dans les processus de professionnalisation.

\section{Former les enseignants de demain}

Le débat sur la formation des maîtres semble souvent porter à l'excès le poids des représentations traditionnelles du système éducatif, et peine à intégrer une vision prospective des évolutions prévisibles. L'article qui rend compte de la formation systématique aux TICE proposée en Corée interpelle de ce point de vue les autres pays : la préparation des maîtres prend-elle suffisamment en compte les nouveaux modèles professionnels liés au développement rapide des TICE et des environnements numériques de travail ?

Ces nouveaux outils modifient le positionnement des enseignants par rapport aux élèves. Leur rôle est désormais moins celui de dispensateurs de savoirs. Face au tableau numérique interactif, ils se retrouvent plutôt à côté des élèves, et en situation de les accompagner dans la démarche de compréhension et d'acquisition de ces savoirs. En cela, les TICE participent de la même évolution que les temps d'accompagnement personnalisé qui s'introduisent aujourd'hui, au fil des réformes, dans tous les secteurs du système éducatif, et qui visent à inciter l'enseignant à prendre en charge plus individuellement les élèves pour les guider dans le processus d'apprentissage. De la même façon les environnements numériques de travail ouvrent de nouveaux temps et de nouveaux modes d'échanges entre les enseignants, les élèves, les familles. La relation pédagogique dépend de moins en moins des lieux consacrés (la classe, le centre de documentation) et des plages réservées dans l'emploi du temps pour se développer plus souplement, en dehors même du temps et de l'espace scolaires. Former les enseignants de demain, c'est tenter de prendre la mesure des déplacements et des recompositions qu'introduira ce nouveau contexte dans les choix didactiques et les pratiques pédagogiques. 
Dans un autre domaine, on constate que le système français reste très marqué par l'opposition du premier et du second degré, dont l'article d'E. Fraisse rappelle les origines. La création des IUFM n'a pas permis d'effacer cette ligne de fracture, et aujourd'hui encore, malgré la réforme en cours, l'investissement des universités n'est pas de même nature selon qu'il s'agit de former les maîtres du primaire et du secondaire. Pourtant, si la tripartition traditionnelle primaire/ secondaire/supérieur garde une forte valeur symbolique, les signes se multiplient qui suggèrent qu'elle est largement dépassée et que tend à s'imposer une nouvelle architecture du système éducatif. Logiquement en effet, l'allongement de la scolarité jusque dans l'enseignement supérieur déplace les frontières, et les dynamiques du système éducatif conduisent plutôt à souligner la cohérence d'une école de base correspondant à la scolarité obligatoire (premier degré + collège), organisée autour des objectifs du socle commun. Lui succède le segment qui va de «bac -3 » à " bac +3 », et qui tend à rapprocher les lycées et le premier cycle de l'enseignement supérieur : c'est tout le sens des réformes en cours (nouvelle organisation des lycées, " cordées de la réussite ", " plan licence ", etc.). Enfin le niveau master et doctorat articule plus étroitement enseignement et recherche.

Cette évolution devrait conduire à aborder sur des bases nouvelles plusieurs questions essentielles pour la formation des enseignants. Par exemple, les professeurs de collège ne devraient-ils pas être formés plutôt dans une logique de cohérence avec le premier degré que sur le modèle de l'enseignant traditionnel du second degré ? Cela pose notamment la question, sinon de la polyvalence, du moins d'une définition moins spécialisée de certains champs disciplinaires. De même la question de l'agrégation pourrait être reposée dans ce cadre. On sait qu'historiquement les CAPES sont des formations secondes par rapport aux agrégations. La «masterisation » récente a renversé cette logique, au point que le statut et l'attractivité de l'agrégation comme concours de recrutement initial deviennent fort incertains : pourquoi ne pas lui donner explicitement comme mission de préparer des enseignants ayant vocation à exercer de part et d'autre du baccalauréat, en lycée et dans le premier cycle de l'enseignement supérieur?

En France comme dans la plupart des pays, la formation des maîtres tente de s'adapter à des enjeux nouveaux. Le processus est en cours, et sur de nombreux sujets les points d'équilibre ne sont pas encore assurés. La responsabilité de l'Université est en tout cas essentielle. Conformément à sa vocation, elle devra articuler formation et recherche pour définir, autour des parcours préparant aux métiers de l'enseignement, des projets didactiques cohérents. Elle devra penser la professionnalisation en termes non réducteurs, en évitant les risques de dérive utilitariste signalés dans nombre d'expériences étrangères : en un mot, elle devra retrouver le rôle qui a été le sien aux origines de l'école républicaine. 